\title{
Chemical composition and evolution of irregular and blue compact galaxies. The dawn of a thirty year journey
}

\author{
M. Tosi \\ INAF - Osservatorio Astronomico di Bologna, via Ranzani 1, 40127 Bologna, Italy \\ e-mail: monica.tosi@oabo.inaf.it
}

The paper by J. Lequeux, M. Peimbert, J. Rayo, A. Serrano, and Silvia Torres-Peimbert (hereafter, LPRST) brings us back to the dawn of the studies on the evolution of late-type dwarfs and of galaxies in general. Thirty years ago, when they wrote their paper, a number of milestones had already been published on the chemical evolution of galaxies (e.g. Salpeter 1955; Schmidt 1959; Tinsley 1968; Cameron \& Truran 1971; Talbot \& Arnett 1971; Lynden-Bell 1975; Audouze \& Tinsley 1976; Mayor 1976; Vigroux et al. 1976, online), but none of them dealt explicitly with late-type dwarf galaxies. Lequeux et al. (1979) set the stage for all subsequent studies of the evolution of irregular and blue compact dwarfs (BCDs). The term pioneer is often used (and sometimes misused) in the astronomical literature. In their case, pioneers is really what they were.

The aims of their study were manifold and quite challenging for the time: a) derive the primeval abundance of helium by extrapolating the $(\mathrm{He} / \mathrm{H})$ vs $(\mathrm{O} / \mathrm{H})$ relation to zero oxygen abundance; b) estimate the primary fraction of ${ }^{14} \mathrm{~N}$ by studying the $(\mathrm{N} / \mathrm{O})$ vs $(\mathrm{O} / \mathrm{H})$ relation; $\mathrm{c})$ derive the heavy element content vs the total galactic mass relation; d) derive the heavy element yield $p(Z)$ by means of the $(\mathrm{O} / \mathrm{H})$ vs. $M_{\mathrm{gas}} / M_{\mathrm{tot}}$ relation; e) put limits on the evolutionary properties of irregulars and BCDs by comparing the results of the previous items with the predictions of chemical evolution models. Quite an ambitious plan, considering the number of papers devoted to each of these topics in the last decades. "Everything you always wanted to know about dwarf galaxies, but were afraid to ask", Woody Allen would have said...

It is impressive to see how valid the results they achieved were, in spite of the poor tools available at the time, but isn't this exactly what pioneers do?! Consider, for instance, their analysis of the nitrogen evolution, aimed at checking the revolutionary suggestion of a partial primary origin of nitrogen done by Edmunds \& Pagel (1978) and Alloin et al. (1979) on the basis of observations of Galactic and extragalactic HII regions. To this aim, LPRST observed the HII regions in six late-type dwarfs and computed chemical evolution models to compare the resulting abundances. Their chemical evolution models were run assuming the instantaneous recycling approximation, an oversimplifying assumption (as they were aware of), especially for an element mostly produced by intermediate-mass, relatively longlived stars. No appropriate tables with nucleosynthesis yields for low and intermediate mass stars had been published yet (the Renzini \& Voli 1981, paper, another of the A\&A most cited ones, was still to come), Arnett (1978) had just published his fundamental paper on massive star yields and the Chiosi \& Caimmi (1979) version with mass loss was just about to appear. Yet, Lequeux et al. were able to put limits on the fraction of primary nitrogen, later confirmed by models computed when stellar lifetimes and appropriate yields could be taken into account (e.g. Matteucci \& Tosi 1985; Diaz \& Tosi 1986, online).

On the primordial helium abundance derived from the extrapolation of the HII region abundances in metal-poor galaxies, the debate has been quite hot for decades between the groups who favored a low value close to $Y_{\mathrm{p}}=0.23$, similar to the one obtained by LPRST (e.g. Pagel et al. 1992; Olive et al. 1997; Peimbert et al. 2000, and references therein), and those who favored a value higher than 0.24 (e.g. Izotov et al. 1994, online). Only the advent of the deus ex-machina WMAP and its incredibly tight constraints on the products of Big Bang nucleosynthesis (Spergel et al. 2003) has ended the quarrel and induced people to converge on $Y_{\mathrm{p}}=0.248$ (Izotov et al. 2007; Peimbert et al. 2007).

Another aspect that, in my opinion, made this paper so interesting for the readers, and still makes it among the most cited ones in the whole lifetime of A\&A, is the combination of good observational data, interpretation tools, and theoretical modeling: a template that authors should always try to adopt. Equally excellent was the choice of the target galaxies, three BCDs (I Zw 18, II Zw 40, and I Zw 70), and three irregulars (NGC 4449, NGC 6822, and IC 10), objects that have become the pet galaxies of many of us, thanks to their intriguing aspects and importance for trying to understand how dwarfs evolve and what the differences and similarities among their various types are.

LPRST correctly admitted that their analysis could not establish whether BCDs have star formation (SF) histories significantly different from those of dwarf irregulars, but they felt that the only real difference is in the present SF rate. This is indeed what we think today (e.g. Tosi 2009, and references therein). They also favored the hypothesis that neither BCDs nor irregulars are young systems and that they all contain old stellar populations. We can now tell that they got it right: all the late-type dwarfs observed at telescopes with enough power 
and resolution to measure their individual stars have turned out to contain objects as old as the lookback time reached by the photometry (e.g. Tosi 2009, and references therein). For instance, NGC 6822 is close enough to allow detection of RR Lyrae variables (Clementini et al. 2003); i.e., stars 10 Gyr old, IC 10, NGC 4449, and IZw18 are too far away to let their horizontal branch stars be visible with current instruments, but HST has shown that they contain red giants (Holtzman et al. 2006; Aloisi et al. 2007; Annibali et al. 2008), i.e. stars at least 2 Gyr old. No late-type dwarf without stars as old as the lookback time has been found yet.

Finally, as far as I am aware, LPRST were the first to discover a relation between the mass and the metallicity of galaxies, which is probably the primary reason for the success of their paper. This relation is clearly a key element in understanding how galaxies evolve, and it has been subsequently confirmed to hold not only for larger samples of dwarf galaxies (e.g. Lee et al. 2006, online), but also for the 53000 star-forming, more massive galaxies measured by the Sloan Digital Sky Survey (Tremonti et al. 2004). In practice, what LPRST found for their 6 dwarfs actually applies to all star-forming galaxies in a 5 orderof-magnitude mass range. Quite a remarkable achievement, indeed: no wonder people keep citing this seminal paper!

To conclude, I think it is instructive to have an idea of what the authors recall of their work. Here is James Lequeux' report: "The history is very simple. Since 1974-75, the Mexican group and myself became increasingly interested in the chemical evolution of galaxies, so that we decided that we should collaborate rather than working separately. We set-up a program of observations which were done by the Mexican side, and I was invited by UNAM to spend 3 months in Mexico where we worked on these data with much enthusiam, in a very friendly atmosphere. I worked hard, but spent most of the week-ends visiting colonial towns and archeological sites which abound around Mexico-City. This stay was one of the most enjoyable periods of my life. On the scientific side, I remember having been much inspired by the famous paper by Larson $\mathcal{F}$ Tinsley (1978), a real landmark in the field although they discussed only the colors of the galaxies. But we did not understand the reason for the relation metallicity/mass of the galaxies that we found. Thus, I was extremely pleased when I saw the paper by Gerola et al. (1980), which gave a very convincing explanation from their model of stochastic self-propagating star formation."

Not only are they excellent scientists and pioneers, but they also show us how fruitful collaborating is and, most important of all, how to enjoy work and life. We should really learn their lesson!

\section{References}

Alloin, D., Collin-Souffrin, S., Joly, M., \& Vigroux, L. 1979, A\&A, 78, 200

Aloisi, A., Clementini, G., Tosi, M., et al. 2007, ApJ, 667, L151

Annibali, F., Aloisi, A., Mack, J., et al. 2008, AJ, 135, 1900

Arnett, W. D. 1978, ApJ, 219, 1008

Audouze, J., \& Tinsley, B. M. 1976, ARA\&A, 14, 43

Cameron, A. G. W., \& Truran, J. W. 1971, JRASC, 65, 1

Chiosi, C., \& Caimmi, R. 1979, A\&A, 80, 234

Clementini, G., Held, E. V., Baldacci, L., \& Rizzi, L. 2003, ApJ, 588, L85

Diaz, A. I., \& Tosi, M. 1986, A\&A, 158, 60

Edmunds, M. G., \& Pagel, B. E. J. 1978, MNRAS, 185, 77P

Gerola, H., Seiden, P. E., \& Schulman, L. S. 1980, ApJ, 242, 517

Holtzman, J. A., Afonso, C., \& Dolphin, A. 2006, ApJS, 166, 534

Izotov, Y. I., Thuan, T. X., \& Lipovetsky, V. A. 1994, ApJ, 435, 647

Izotov, Y. I., Thuan, T. X., \& Stasińska, G. 2007, ApJ, 662, 15

Larson, R. B., \& Tinsley, B. M. 1978, ApJ, 219, 46

Lee, H., Skillman, E. D., Cannon, J. M., et al. 2006, ApJ, 647, 970

Lequeux, J., Peimbert, M., Rayo, J. F., Serrano, A., \& Torres-Peimbert, S. 1979, A\&A, 80, 155

Lynden-Bell, D. 1975, Vistas Astron., 19, 299

Matteucci, F., \& Tosi, M. 1985, MNRAS, 217, 391

Mayor, M. 1976, A\&A, 48, 301

Olive, K. A., Steigman, G., \& Skillman, E. D. 1997, ApJ, 483, 788

Pagel, B. E. J., Simonson, E. A., Terlevich, R. J., \& Edmunds, M. G. 1992, MNRAS, 255, 325

Peimbert, M., Peimbert, A., \& Ruiz, M. T. 2000, ApJ, 541, 688

Peimbert, M., Luridiana, V., \& Peimbert, A. 2007, ApJ, 666, 636

Renzini, A., \& Voli, M. 1981, A\&A, 94, 175

Salpeter, E. E. 1955, ApJ, 121, 161

Schmidt, M. 1959, ApJ, 129, 243

Spergel, D. N., Verde, L., Peiris, H. V., et al. 2003, ApJS, 148, 175

Talbot, Jr., R. J., \& Arnett, W. D. 1971, ApJ, 170, 409

Tinsley, B. M. 1968, ApJ, 151, 547

Tosi, M. 2009, in IAU Symp., 258 [arXiv: 0901. 1090]

Tremonti, C. A., Heckman, T. M., Kauffmann, G., et al. 2004, ApJ, 613, 898

Vigroux, L., Audouze, J., \& Lequeux, J. 1976, A\&A, 52, 1 